\title{
Clonal spread of antimicrobial-resistant Escherichia coli isolates among pups in two kennels
}

\author{
Kazuki Harada*, Erika Morimoto, Yasushi Kataoka, Toshio Takahashi
}

\begin{abstract}
Although the dog breeding industry is common in many countries, the presence of antimicrobial resistant bacteria among pups in kennels has been infrequently investigated. This study was conducted to better understand the epidemiology of antimicrobial-resistant Escherichia coli isolates from kennel pups not treated with antimicrobials. We investigated susceptibilities to 11 antimicrobials, and prevalence of extended-spectrum $\beta$-lactamase (ESBL) in 86 faecal E. coli isolates from 43 pups in two kennels. Genetic relatedness among all isolates was assessed using pulsed-field gel electrophoresis (PFGE). Susceptibility tests revealed that $76 \%$ of the isolates were resistant to one or more of tested antimicrobials, with resistance to dihydrostreptomycin most frequently encountered (66.3\%) followed by ampicillin (60.5\%), trimethoprim-sulfamethoxazole (41.9\%), oxytetracycline (26.7\%), and chloramphenicol (26.7\%). Multidrug resistance, defined as resistance against two or more classes of antimicrobials, was observed in 52 (60.5\%) isolates. Three pups in one kennel harboured SHV-12 ESBL-producing isolates. A comparison between the two kennels showed that frequencies of resistance against seven antimicrobials and the variation in resistant phenotypes differed significantly. Analysis by PFGE revealed that clone sharing rates among pups of the same litters were not significantly different in both kennels (64.0\% vs. 88.9\%), whereas the rates among pups from different litters were significantly different between the two kennels $(72.0 \%$ vs. 33.3\%, $P<0.05)$. The pups in the two kennels had antimicrobial-resistant E. coli clones, including multidrug-resistant and ESBL-producing clones. It is likely that resistant and susceptible bacteria can clonally spread among the same and/or different litters thus affecting the resistance prevalence.
\end{abstract}

\section{Findings}

Spread of antimicrobial-resistant bacteria from companion animals to humans causes concern but the role of companion animals as reservoirs of antimicrobial resistant bacteria requires further investigation [1]. Escherichia coli are commonly found in the intestinal tract of animals, including dogs, and constitute a reservoir of resistant genes for potentially pathogenic bacteria [1] Dogs may also be a reservoir of $E$. coli strains that cause extraintestinal infections in humans [2]. Antimicrobial resistance of canine $E$. coli has previously been investigated [3-5].

Antimicrobial-resistant E. coli have been isolated more frequently in kennel dogs than in individually owned dogs [3]. Breeding of multiple dogs at one location may increase the risk of spreading antimicrobial resistant

\footnotetext{
* Correspondence: k-harada@nvlu.ac.jp

Laboratory of Veterinary Microbiology, Nippon Veterinary and Life Science University, 1-7-1, Kyonan-cho, Musashino, Tokyo 180-8602, Japan
}

clones in the population similar to livestock on the same premises [6,7].

The purpose of the present study was to compare phenotypic and genetic characteristics of antimicrobial resistant $E$. coli isolated from the faeces of pups in kennels, and to investigate genetic relatedness among these isolates as the epidemiology of antimicrobial resistant bacteria in dog populations has not been extensively studied.

Faecal samples were obtained from 43 apparently healthy pups not above two months of age from two kennels ( $\mathrm{A}$ and $\mathrm{B})$. Twenty-five pups were from eight litters in kennel A (A-a to A-h), and 18 were from five litters in kennel B (B-a to B-e; Table 1). There was no history of antimicrobial use in the pups but the dams may have been administered lincomycin for postpartum infection prophylaxis. Briefly, faecal swabs were plated onto desoxycholate-hydrogen sulphide-lactose agar (Eiken Chemical, Tochigi, Japan) and incubated overnight at $37^{\circ} \mathrm{C}$ in an aerobic atmosphere. Subsequently, 
Table 1 Details of surveyed pups in this study

\begin{tabular}{|c|c|c|c|c|c|c|}
\hline Kennel & Litter & Breed & No. of pups & Pup identity & Date of birth & Age in days \\
\hline \multirow[t]{9}{*}{$A$} & $A-a$ & Toy poodle & 3 & $A-a-1-A-a-3$ & $5 / 13 / 2009$ & 30 \\
\hline & $A-b$ & Toy poodle & 3 & $A-b-1-A-b-3$ & $5 / 15 / 2009$ & 28 \\
\hline & $A-C$ & Chihuahua & 2 & $A-C-1-A-C-2$ & 4/17/2009 & 57 \\
\hline & $A-d$ & Toy poodle & 3 & $A-d-1-A-d-3$ & $6 / 23 / 2009$ & 45 \\
\hline & A-e & Toy poodle & 4 & A-e-1 - A-e-4 & $7 / 1 / 2009$ & 37 \\
\hline & A-f & Toy poodle & 5 & $A-f-1-A-f-5$ & $7 / 9 / 2009$ & 29 \\
\hline & $A-g$ & Toy poodle & 3 & $A-g-1-A-g-3$ & $9 / 2 / 2009$ & 38 \\
\hline & A-h & Toy poodle & 2 & A-h-1 - A-h-2 & $9 / 6 / 2009$ & 35 \\
\hline & Total & & 25 & & & \\
\hline \multirow[t]{6}{*}{$\bar{B}$} & B-a & Maltese & 2 & B-a-1 - B-a-2 & $8 / 3 / 2009$ & 46 \\
\hline & $B-b$ & Beagle & 4 & B-b-1 - B-b-4 & $7 / 29 / 2009$ & 51 \\
\hline & $B-C$ & Miniature dachshund & 5 & $B-C-1-B-C-5$ & $8 / 1 / 2009$ & 51 \\
\hline & $B-d$ & Papillon & 3 & $B-d-1-B-d-3$ & 8/17/2009 & 53 \\
\hline & B-e & Chihuahua & 4 & B-e-1 - B-e-4 & $8 / 25 / 2009$ & 55 \\
\hline & Total & & 18 & & & \\
\hline
\end{tabular}

two lactose fermenting colonies with typical E. coli morphology were picked and subjected to confirmatory Gram staining, indole, methyl red, Voges-Proskauer, and Simmons' Citrate tests.

Informed consent from both kennels was obtained and treated in accordance with the Japanese Law Concerning the Protection of Personal Information (Law No. 57, 2003).

Minimum inhibitory concentrations of ampicillin (AMP), cefazolin, ceftiofur, dihydrostreptomycin (DHS), gentamicin, kanamycin, oxytetracycline (OTC), chloramphenicol (CHL), trimethoprim-sulfamethoxazole (SXT), nalidixic acid and enrofloxacin were determined using the agar dilution method according to the Clinical and Laboratory Standards Institute (CLSI) Guidelines [8]. CLSI resistance breakpoints [8,9] were used in the categorical analysis of all drugs except DHS where $32 \mu \mathrm{g} /$ $\mathrm{mL}$ was used as reported elsewhere [10]. For quality control, E. coli ATCC 25922 was used.

All isolates were screened for extended-spectrum $\beta$ lactamase $(\mathrm{ESBL})$ production by the combination disk test (cefotaxime and ceftazidime with or without clavulanic acid) [8]. ESBL-producing strains were examined for $\beta$-lactamase-encoding genes including blaTEM, blaSHV, blaCTX-M-2 group, and blaCTX-M-9 group by polymerase chain reaction and sequencing [11].

Pulsed-field gel electrophoresis (PFGE) was performed according to standard methods outlined by PulseNet [12]. DNA was digested with XbaI (Takara, Shiga, Japan) and electrophoresed on a CHEF DRII (Bio-Rad Laboratories, Hercules, CA, USA), with switch times of 2.2-54.2 $\mathrm{s}$ at $14^{\circ} \mathrm{C}$ for $20 \mathrm{~h}$. PFGE profiles were analysed using BioNumerics software version 4.0 (Applied Maths, TX, USA). DNA fragments on each gel were normalised using the $\lambda$ molecular weight marker on each gel to enable comparisons between different gels. Cluster analysis was performed by the unweighted pair group method using arithmetic average. DNA relatedness was calculated based on the Dice coefficient.

The prevalence of antimicrobial resistance and rates of clone sharing between the two kennels were compared using Fisher's exact test.

In this study, we investigated the prevalence of antimicrobial resistance in faecal $E$. coli isolates from kennel pups without a history of antimicrobial use; however, their dams may have been administered with lincomycin. If this is the case, the possibility that the pups were exposed to the agent via the milk cannot be excluded. It is generally known that lincomycin is inactive against aerobic Gram-negative bacteria including $E$. coli, but active against Gram-positive and/or anaerobic bacteria [13], possibly causing changes in the intestinal microflora composition. The effects of an altered intestinal flora on the population of resistant and susceptible $E$. coli are unknown, but may need to be taken into account in the present results.

We found that $75.6 \%(n=65)$ of $E$. coli isolates originating from 35 pups in two kennels, were resistant to one or more of the antimicrobials tested. Resistance to DHS was most frequent $(66.3 \%)$, followed by AMP (60.5\%), SXT (41.9\%), OTC (26.7\%), and CHL (26.7\%) (Table 2). Multidrug resistance defined as resistance against two or more classes of antimicrobials was observed in $60.5 \%(n=52)$ of isolates, originating from 29 pups in two kennels. Comparison between kennels A and $B$ revealed that the prevalence of resistance against seven of the tested antimicrobials differed significantly $(P<0.05)$. Twelve and five resistance patterns were observed in kennels A and B, respectively, with the patterns differing between the two kennels, except for the 
Table 2 The minimum inhibitory concentration (MIC) range and resistance rates among Escherichia coli isolates from pups originating from two kennels (A and $B$ )

\begin{tabular}{|c|c|c|c|c|c|c|c|}
\hline \multirow[t]{2}{*}{ Substance $^{a}$} & \multirow[t]{2}{*}{ MIC range $(\mu \mathrm{g} / \mathrm{mL})$} & \multirow[t]{2}{*}{$\mathrm{MIC}_{50}$} & \multirow[t]{2}{*}{$\mathrm{MIC}_{90}$} & \multirow{2}{*}{$\begin{array}{l}\text { Resistance } \\
\text { breakpoints } \\
(\mu \mathrm{g} / \mathrm{mL})^{\mathrm{b}}\end{array}$} & \multicolumn{3}{|c|}{$\begin{array}{l}\text { No. of resistant isolates (\%)/No. of pups } \\
\text { that harboured resistant isolate(s) (\%) }\end{array}$} \\
\hline & & & & & $\begin{array}{l}\text { Total } \\
(\mathrm{n}=86 / 43)\end{array}$ & $\begin{array}{l}\text { Kennel A } \\
(\mathrm{n}=50 / 25)\end{array}$ & $\begin{array}{l}\text { Kennel B } \\
(\mathrm{n}=36 / 18)\end{array}$ \\
\hline AMP & $2->512$ & $>512$ & $>512$ & $\geq 32$ & $52(60.5) / 30(69.8)$ & $29(58.0) / 17$ (68.0) & $23(63.9) / 13(72.2)$ \\
\hline CFZ & $8-128$ & 4 & 8 & $\geq 32$ & $5(5.8) / 3(7.0)$ & $0(0) / 0(0)$ & $5(13.9)^{*} / 3(16.7)$ \\
\hline CEF & $\leq 0.125-32$ & 0.5 & 1 & $\geq 8$ & $5(5.8) / 3(7.0)$ & $0(0) / 0(0)$ & $5(13.9)^{*} / 3(16.7)$ \\
\hline DHS & $2->512$ & 512 & $>512$ & $\geq 32$ & $57(66.3) / 32(74.4)$ & $35(70.0) / 19(76.0)$ & $22(61.1) / 13(72.2)$ \\
\hline GEN & $0.5-256$ & 1 & 128 & $\geq 16$ & $16(18.6) / 12(27.9)$ & $16(32.0)^{*} / 12(48.0)^{*}$ & $0(0) / 0(0)$ \\
\hline KAN & $2->512$ & 4 & 16 & $\geq 64$ & $3(3.5) / 2(4.7)$ & $3(6.0) / 2(8.0)$ & $0(0) / 0(0)$ \\
\hline OTC & $1-512$ & 2 & 512 & $\geq 16$ & $23(26.7) / 17$ (39.5) & $18(36.0)^{*} / 14(56.0)^{*}$ & $5(13.9) / 3(16.7)$ \\
\hline $\mathrm{CHL}$ & $4->512$ & 8 & 512 & $\geq 32$ & $23(26.7) / 17(39.5)$ & $18(36.0)^{*} / 14(56.0)^{*}$ & $5(13.9) / 3(16.7)$ \\
\hline NAL & $2->512$ & 4 & 16 & $\geq 32$ & $7(8.1) / 5(11.6)$ & $2(4.0) / 2(8.0)$ & $5(13.9) / 3(16.7)$ \\
\hline ENR & $\leq 0.03-256$ & 0.06 & 1 & $\geq 4$ & $5(5.8) / 3(7.0)$ & $0(0) / 0(0)$ & $5(13.9)^{*} / 3(16.7)$ \\
\hline SXT & $\leq 0.25 / 4.75->64 / 1216$ & $1 / 19$ & $>64 / 1216$ & $\geq 16 / 304$ & $36(41.9) / 21(48.8)$ & $27(54.0)^{*} / 16(64.0)^{*}$ & $9(25.0) / 5(27.8)$ \\
\hline
\end{tabular}

${ }^{a} A M P$, ampicillin; CFZ, cefazolin; CEF, ceftiofur; DHS, dihydrostreptomycin; GEN, gentamicin; KAN, kanamycin; OTC, oxytetracycline; CHL, chloramphenicol; NAL, nalidixic acid; ENR, enrofloxacin; SXT, trimethoprim-sulfamethoxazole.

${ }^{b}$ The breakpoints for AMP, CFZ, CEF, GEN, KAN, OTC, CHL, ENR and SXT, and that for NAL were based on CLSI document M31-A3 [9] and M100-S20 [8], respectively, whereas the breakpoint of DHS was based on an epidemiological cut-off value according to another report [10].

AMP-DHS-SXT and DHS resistance phenotypes (Table 3). These findings indicate that the prevalence of antimicrobial resistant $E$. coli in pups varies between kennels.

Additionally, the XbaI-digested PFGE revealed 13 and 10 distinct major profiles ( $\geq 90 \%$ Dice similarity) in 50 and 36 isolates from 25 and 18 pups from kennels A and $\mathrm{B}$ (Figures 1 and 2), respectively. These PFGE profiles correlated highly with resistance phenotypes, except for profiles A-1, A-5 and A-11. Of all 43 pups, 17 pups harboured two isolates differentiated by PFGE and/or resistance phenotypic profiles, indicating that diverse $E$. coli populations can colonise intestinal flora during infancy. Sixteen of 25 pups in kennel A (i.e. two, three, three, five, and three pups within litters A-a, A-b, A-d, A-f, and A-g, respectively) and 16 of 18 pups in kennel B (i.e. two, four, four, two, and four pups within from

Table 3 The distribution of resistance phenotypes among Escherichiacoli isolates from pups in two kennels (A and B)

\begin{tabular}{|c|c|c|c|}
\hline \multirow[t]{3}{*}{ Resistance patterns $^{\mathrm{a}}$} & \multicolumn{3}{|c|}{ No. of resistant isolates (\%)/No. of pups that harboured resistant isolate (s) (\%) } \\
\hline & Total & Kennel A & Kennel B \\
\hline & $(n=86 / 43)$ & $(n=50 / 25)$ & $(n=36 / 18)$ \\
\hline AMP-CFZ-CFT-OTC-CHL-NAL-ENR & $5(5.8) / 3(7.0)$ & $0(0) / 0(0)$ & $5(13.9) / 3(16.7)$ \\
\hline AMP-DHS-GEN-KAN-OTC-CHL-SXT & $3(3.5) / 2(4.7)$ & $3(6.0) / 2(8.0)$ & $0(0) / 0(0)$ \\
\hline AMP-DHS-GEN-OTC-CHL-NAL-SXT & $1(1.2) / 1(2.3)$ & $1(2.0) / 1(4.0)$ & $0(0) / 0(0)$ \\
\hline AMP-DHS-GEN-OTC-CHL-SXT & $11(12.8) / 9(20.9)$ & $11(22.0) / 9(36.0)$ & $0(0) / 0(0)$ \\
\hline AMP-DHS-GEN-CHL-SXT & $1(1.2) / 1(2.3)$ & $1(2.0) / 1(4.0)$ & $0(0) / 0(0)$ \\
\hline AMP-DHS-OTC-SXT & $2(2.3) / 1(2.3)$ & $2(4.0) / 1(4.0)$ & $0(0) / 0(0)$ \\
\hline AMP-DHS-CHL-SXT & $1(1.2) / 1(2.3)$ & $1(2.0) / 1(4.0)$ & $0(0) / 0(0)$ \\
\hline AMP-DHS-SXT & $16(18.6) / 13(30.2)$ & $8(16.0) / 8(32.0)$ & $8(22.2) / 5$ (27.8) \\
\hline AMP-DHS-CHL & $1(1.2) / 1(2.3)$ & $1(2.0) / 1(4.0)$ & $0(0) / 0(0)$ \\
\hline AMP-DHS & $10(11.6) / 6(14.0)$ & $0(0) / 0(0)$ & $10(27.8) / 6(33.3)$ \\
\hline DHS-SXT & $1(1.2) / 1(2.3)$ & $0(0) / 0(0)$ & $1(2.8) / 1(5.6)$ \\
\hline AMP & $1(1.2) / 1(2.3)$ & $1(2.0) / 1(4.0)$ & $0(0) / 0(0)$ \\
\hline DHS & $10(11.6) / 7(16.3)$ & $7(14.0) / 5(20.0)$ & $3(8.3) / 2(11.1)$ \\
\hline OTC & $1(1.2) / 1(2.3)$ & $1(2.0) / 1(4.0)$ & $0(0) / 0(0)$ \\
\hline NAL & $1(1.2) / 1(2.3)$ & $1(2.0) / 1(4.0)$ & $0(0) / 0(0)$ \\
\hline Susceptible & $21(24.4) / 13(30.2)$ & $12(24.0) / 7(28.0)$ & $9(25.0) / 6(33.3)$ \\
\hline
\end{tabular}

${ }^{\mathrm{a} A M P}$, ampicillin; CFZ, cefazolin; CEF, ceftiofur; DHS, dihydrostreptomycin; GEN, gentamicin; KAN, kanamycin; OTC, oxytetracycline; CHL, chloramphenicol; NAL, nalidixic acid; ENR, enrofloxacin; SXT, trimethoprim-sulfamethoxazole. 


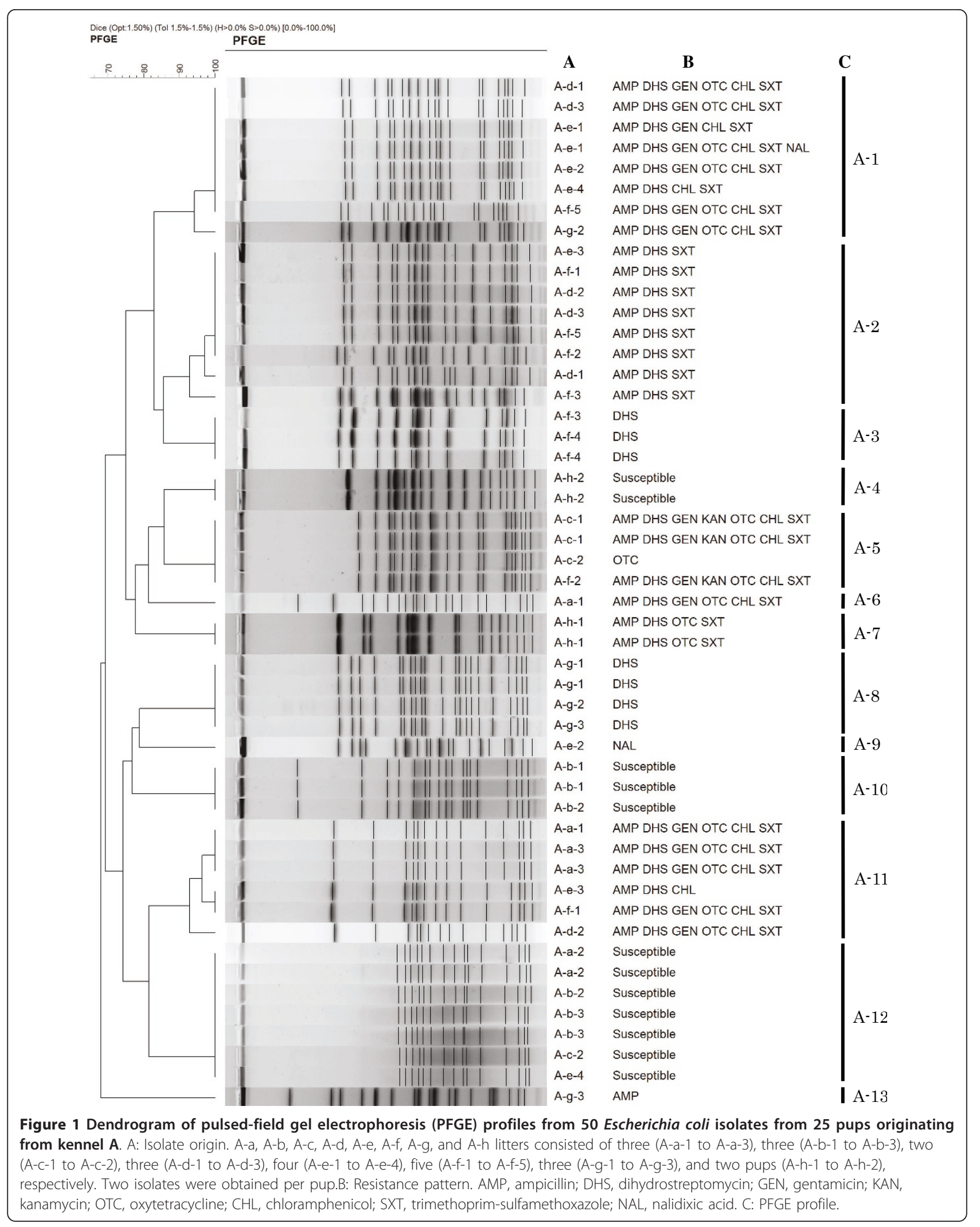




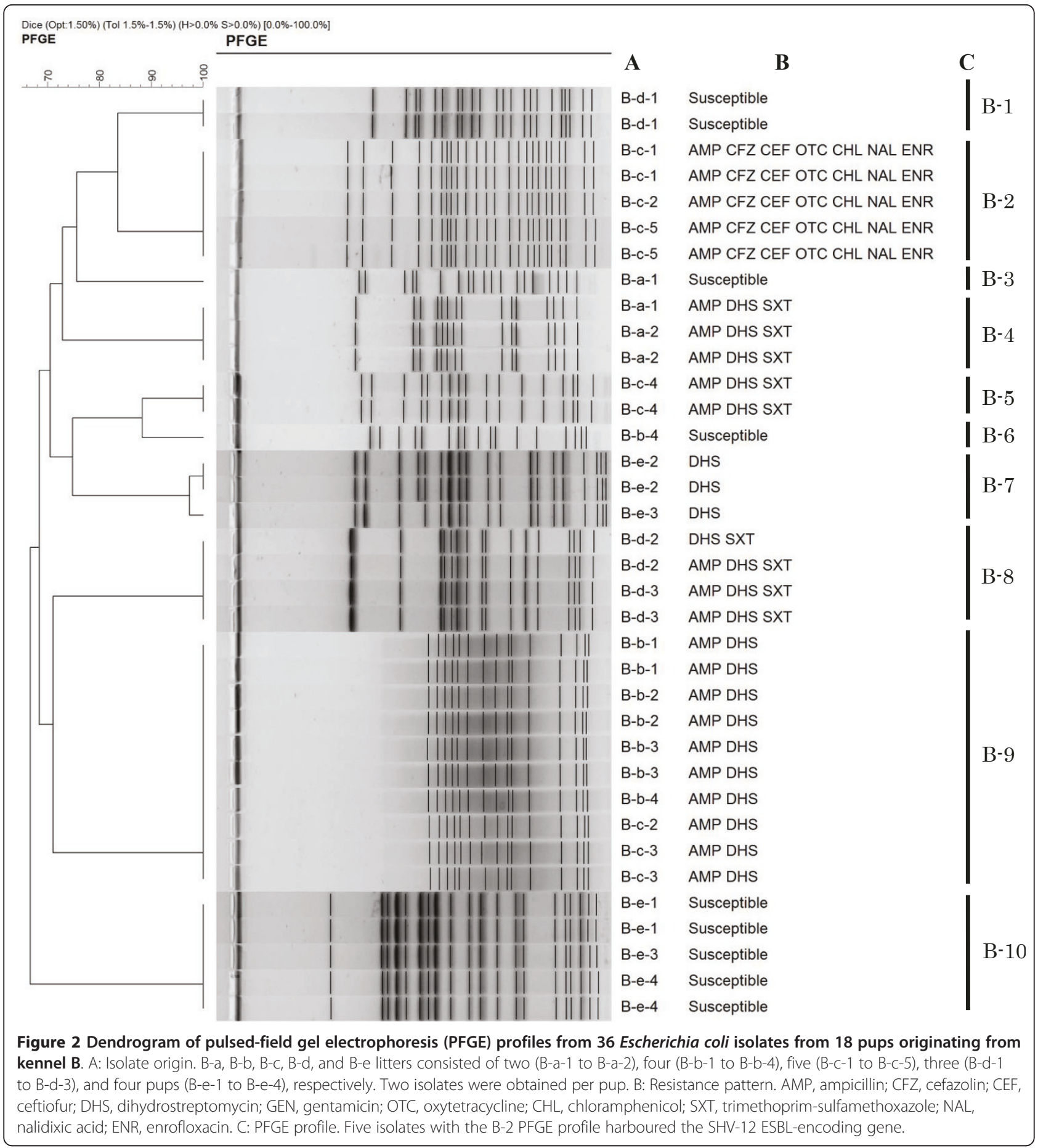

litters B-a to B-e, respectively) shared at least one E. coli clone, defined as an isolate with identical PFGE and resistance phenotypic profiles, with one or more pups of the same litters. There was no significant difference in the clone sharing rates within the same litters between the two kennels. In the two kennels, all pups of the same litters, and their respective dams, were raised together in one cage, implying that E. coli may be transmitted horizontally via faeces. Another possibility may be vertical transfer from mothers via their milk and vaginal flora [14]. These data suggest that pups from the same litter are likely to be exposed to common sources of $E$. coli resulting in clonal spread of organisms, including antimicrobial resistant isolates. 
The following 24 pups in the two kennels shared at least one E. coli clone (i.e. the clones harbouring PFGE profiles A-1, 2, 5, 11, 12, and B-9) among the different litters; three, two, two, three, three, four, and one pups of from litters A-a to A-g in kennel A, and four and two pups of litters B-b and B-c, respectively. These litters were temporally separated (8-83 days) and originated from different mothers without direct contact, suggesting that $E$. coli clones may have originated from a persistent external source. One possibility, as suggested by other studies, is that the pups acquired $E$. coli from their human contacts $[15,16]$. Unlike clone sharing rates among the same litters, the rates among different litters were significantly different between kennels A and B [18/25 (72.0\%) vs. 6/18 (33.3\%) pups, respectively, $P<0.05]$. This finding suggests that clone sharing rates among different litters can vary between kennels. Further study is needed to clarify the potential transmission route(s) between kennel pups. Overall, our data indicates that clonal spread of $E$. coli plays an important role in acquisition of resistant isolates by kennel pups.

The prevalence of ESBL-producing isolates in companion animals and their potential impact on human health is a major issue [17]. In the present study, the SHV-12 ESBL-encoding gene was detected in five isolates (5.8\%), exhibiting identical PFGE and resistance phenotypic profiles, from three pups within a litter (Figure 2). The reason for the occurrence of these resistant isolates was not apparent. To the best of our knowledge, this is the first time that SHV-12 $\beta$-lactamase has been detected in $E$. coli of canine origin in Japan, although it has been previously reported in other countries $[18,19]$. The present findings suggest that attention needs to be paid to dogs as a potential reservoir of ESBL-producing E. coli isolates in Japan.

In conclusion, our data show that pups in kennels can harbour multidrug-resistant $E$. coli isolates, including ESBL-producing isolates. The present results also indicate that resistant and susceptible $E$. coli isolates can clonally spread not only within the same litter but also among different litters thus affecting the prevalence of resistant organisms in a kennel. Further studies are needed to fully understand the epidemiological spread of antimicrobial resistant bacteria among pups in kennels.

\section{Acknowledgements}

The authors wish to acknowledge Dr. Shiki Kawabe for providing faecal swabs from pups. This work was financially supported by a grant from the Ministry of Education, Culture, Sports, Science and Technology, Japan (Grantin-Aid for Research Activity Start-up, No. 21880043).

\section{Authors' contributions}

$\mathrm{KH}$ and EM carried out antimicrobial susceptibility testing, $\mathrm{PCR}$, sequencing, and PFGE. KH analysed the data. $\mathrm{KH}, \mathrm{YK}$ and $\Pi$ were involved in the study design and preparation of the manuscript. $\mathrm{KH}$ drafted the manuscript. All authors read and approved the final manuscript.

\section{Competing interests}

The authors declare that they have no competing interests.

Received: 5 September 2010 Accepted: 17 February 2011 Published: 17 February 2011

\section{References}

1. Guardabassi L, Schwarz S, Lloyd DH: Pet animals as reservoirs of antimicrobial-resistant bacteria. J Antimicrob Chemother 2004, 54:321-332.

2. Johnson JR, Stell AL, Delavari P: Canine feces as a reservoir of extraintestinal pathogenic Escherichia coli. Infect Immun 2001, 69:1306-1314.

3. De Graef EM, Decostere A, Devriese LA, Haesebrouck F: Antibiotic resistance among fecal indicator bacteria from healthy individually owned and kennel dogs. Microb Drug Resist 2004, 10:65-69.

4. Rantala M, Lahti E, Kuhalampi J, Pesonen S, Järvinen AK, SaijonmaaKoulumies, Honkanen-Buzalski T: Antimicrobial resistance in Staphylococcus spp., Escherichia coli and Enterococcus spp. in dogs given antibiotics for chronic dermatological disorders, compared with nontreated control dogs. Acta Vet Scand 2004, 45:37-45.

5. Costa D, Poeta P, Sáenz Y, Coelho AC, Matos M, Vinué L, Rodrigues J, Torres C: Prevalence of antimicrobial resistance and resistance genes in faecal Escherichia coli isolates recovered from healthy pets. Vet Microbiol 2008, 127:97-105.

6. Hoyle DV, Knight HI, Shaw DJ, Hillman K, Pearce MC, Low JC, Gunn GJ, Woolhouse ME: Acquisition and epidemiology of antibiotic-resistant Escherichia coli in a cohort of newborn calves. J Antimicrob Chemother 2004, 53:867-871.

7. Bortolaia V, Bisgaard M, Bojesen AM: Distribution and possible transmission of ampicillin- and nalidixic acid-resistant Escherichia coli within the broiler industry. Vet Microbiol 2010, 142:3-4.

8. Clinical and Laboratory Standards Institute: Performance Standards for Antimicrobial Susceptibility Testing; Twentieth Informational Supplement. CLSI document M100-S20 Wayne, PA; 2010.

9. Clinical and Laboratory Standards Institute: Performance Standards for Antimicrobial Disk and Dilution Susceptibility Tests for Bacteria Isolated From Animals; Approved Standard - Third Edition. CLSI document M31-A3 Wayne, PA; 2008

10. Asai T, Kojima A, Harada K, Ishihara K, Takahashi T, Tamura Y: Correlation between the usage volume of veterinary therapeutic antimicrobials and resistance in Escherichia coli isolated from the feces of food-producing animals in Japan. Jpn J Infect Dis 2005, 58:369-372.

11. Kojima A, Ishii Y, Ishihara K, Esaki H, Asai T, Oda C, Tamura Y, Takahashi T, Yamaguchi K: Extended-spectrum-beta-lactamse-producing Escherichia coli strains isolated from farm animals from 1999 to 2002: report from the Japanese Veterinary Antimicrobial Resistance Monitoring Program. Antimicrob Agents Chemother 2005, 49:3533-3537.

12. Centers for Disease Control and Prevention: One-day (24-48 h) standardization laboratory protocol for molecular sub-typing of Escherichia coli 0157:H7, non-typhoidal Salmonella serotypes, and Shigella sonnei by pulsed-field gel electrophoresis (PFGE). PulseNet PFGE manual National Center for Infectious Diseases, Atlanda, GA; 2004.

13. Spízek J, Rezanka T: Lincomycin, clindamycin and their applications. App/ Microbiol Biotechnol 2004, 64:455-464.

14. Münnich A, Lübke-Becker A: Escherichia coli infections in newborn pups - clinical and epidemiological investigations. Theriogenol 2004, 62:562-575.

15. Sannes MR, Kuskowski MA, Johnson JR: Antimicrobial resistance of Escherichia coli strains isolated from urine of women with cystitis or pyelonephritis and feces of dogs and healthy humans. J Am Vet Med Assoc 2004, 225:368-373.

16. Stenske KA, Bemis DA, Gillespie BE, D'Souza DH, Oliver SP, Draughon FA, Matteson KJ, Bartges JW: Comparison of clonal relatedness and antimicrobial susceptibility of fecal Escherichia coli from healthy dogs and their owners. Am J Vet Res 2009, 70:1108-1116.

17. Li XZ, Mehrotra M, Ghimire S, Adewoye L: Beta-lactam resistance and beta-lactamases in bacteria of animal origin. Vet Microbio/ 2007, 121:197-214. 
18. Briňas $L$, Moreno MA, Teshager $T$, Zarazaga M, Sáenz Y, Porrero C,

Dominguez L, Torres C: Beta-lactamase characterization in Escherichia coli isolates with diminished susceptibility or resistance to extendedspectrum cephalosporins recovered from sick animals in Spain. Microb Drug Resist 2003, 9:201-209.

19. Carattoli A, Lovari S, Franco A, Cordaro G, Di Matteo P, Battisti A: Extendedspectrum beta-lactamases in Escherichia coli isolated from dogs and cats in Rome, Italy, from 2001 to 2003. Antimicrob Agents Chemother 2005, 49:833-835.

doi:10.1186/1751-0147-53-11

Cite this article as: Harada et al: Clonal spread of antimicrobial-resistant Escherichia coli isolates among pups in two kennels. Acta Veterinaria Scandinavica 2011 53:11.

\section{Submit your next manuscript to BioMed Central} and take full advantage of:

- Convenient online submission

- Thorough peer review

- No space constraints or color figure charges

- Immediate publication on acceptance

- Inclusion in PubMed, CAS, Scopus and Google Scholar

- Research which is freely available for redistribution

Submit your manuscript at www.biomedcentral.com/submit 